Anu. Génét. Sél. Anim., I979, 11 (3), 223-240.

\title{
Evaluation des aptitudes laitières des brebis de race pure ou croisées Romanov
}

\author{
J. C. FLAMAN'T et B. BONAITI (*) \\ avec la collaboration technique de \\ J. Hali,auer, Michèle Jacquin, Suzanne Crochez, J. J. Chambeyron
}

\author{
Station d'Amélioration Génétique des Animaux \\ Centre de Recherches agronomiques de Toulouse, I.N.R.A., \\ Auzeville, 3rз2o Castanet Tolosan \\ (*) Station de Génétique quantitative et appliquée, \\ Centre national de Recherches zootechniques, I.N.R.A., \\ 78350 Jouy-en-Josas
}

\begin{abstract}
Résumé
Plusieurs types de comparaisons ont été réalisées pour évaluer le potentiel laitier de la race Romanov.

Les brebis Romanov élevées en race pure réagissent positivement aux sollicitations d'un nombre élevé d'agneaux : la supériorité de production laitière, évaluée par injection d'ocytocine, de brebis allaitant 3 agneaux s'elève à $73 \mathrm{p}$. Ioo par rapport aux brebis allaitant i seul agneau né cependant de portées de 2, 3 ou 4 agneaux. Cet accroissement de production dû à l'augmentation du nombre d'agneaux allaités est comparable à celui observé sur d'autres races.

A nombre d'agneaux allaités égal (2), les brebis croisées Romanov $\times$ Berrichonne $d u$ Cher présentant le pourcentage de sang Romanov le plus élevé (3/4 Romanov) tenđent à manifester les aptitudes laitières les plus faibles bien que le potentiel de croissance des agneaux, évalué en allaitement artificiel ou sur des agneaux nés doubles mais élevés simples sous la mère, soit au moins aussi important que pour les autres génotypes.

L'évaluation des aptitudes laitières des brebis Romanov est effectuée également indépendamment de la tétée de l'agneau à l'aide de la traite mécanique : les performances enregistrées et la persistance moyenne sont nettement inférieures à celles d'un lot témoin de brebis Lacaune conduites sans sélection.

L'influence d'un niveau de production laitière plus faible sur l'élevage des agneaux est discutée dans le cas de différentes stratégies de croisements mettant en œuvre la race Romanov.
\end{abstract}

\section{I. - Introduction}

Le progrès technique en matière de production ovine est marqué au cours de ces 20 dernières années par la recherche d'une prolificité plus élevée et notamment par l'introduction des races du type Romanov et Finnois dont les brebis 
atteignent une taille de portée moyenne proche de 3 agneaux (DJNALD et al., I968; DESVIGNES, I970). Différentes solutions sont étudiées pour réaliser une utilisation raisonnée de ces races (TCHAMITCHIAN et al., I975) : schémas de croisements à double étage, création de nouvelles races comprenant 25 ou $50 \mathrm{p}$. Ioo de gènes Romanov ou Finnois (Mc CLELLAND, I975; RICORDEAU et al., I976), sinon même utilisation des femelles de race pure en croisement simple avec des mâles de races à viande (Tchamitchian, I975). Cette possibilité d'accroître la productivité numérique du cheptel ovin redonne de l'intérêt aux travaux concernant la production laitière des brebis, celle-ci pouvant constituer un facteur limitant pour l'allaitement des agneaux nés et élevés "multiples ».

En effet, comme le faisait déjà remarquer DESvignes (I97I) les performances de croissance des agneaux Romanov purs sont relativement faibles, de l'ordre de I 70 à $200 \mathrm{~g} / \mathrm{j}$ au cours du premier ou des 2 premiers mois (tabl. I). Le caractère limitant de la quantité de lait disponible pour les agneaux de portée multiple est révélé par 1'amélioration des résultats individuels de croissance de 20 à 50 points lorsqu'on a recours à l'allaitement artificiel. Il n'en demeure pas moins que si l'on considère le gain de poids total d'une portée, celui d'une portée triple est supérieur de plus du double à celui d'un seul agneau élevé simple : 2,05 fois pour YADRICHEV (I969) et 2,50 pour Flaman'T et LABUSSİ̀re (I972) dans le cas d'agneaux nés de portées multiples, élevés simples ou triples.

Les premières observations sur la production laitière des brebis Romanov réalisées par FLAMANT et LABUSSIÈRE (I972) donnent des indications sur la réponse

\section{TABLEAU I}

Performances laitières des brebis Romanov en phase d'allaitement et vitesse de croissance des agneaux au cours des 3 premiers mois (vésultats bibliographiques) Milk yield of Romanov ewes in suckling and growth rate of the lambs during the firths three months (bibliography)

\begin{tabular}{c|c|c}
\hline \hline Auteur & Critère mesuré & Performances observées \\
\hline
\end{tabular}

a) Production laitiève $(\mathrm{kg})$

\begin{tabular}{|c|c|c|}
\hline Smiknova (1958). & I oo premiers jours & I I I à I 57 \\
\hline KOVNEREV ct al. (1967) & 20 premiers jours & 50 à 60 \\
\hline MAGOMEDOV (I975a) . & premier mois & $64-68(*)$ \\
\hline MAGOMEDOV (1975a) & 3 premiers mois & I $50-$ I $58\left(^{*}\right)$ \\
\hline
\end{tabular}

b) Vitesse de croissance journalière $(g / j)$

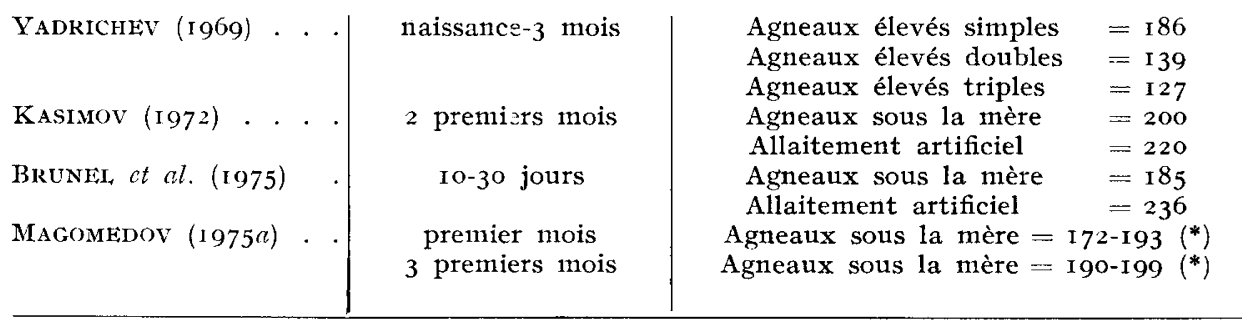

(*) Selon le lot d'animaux considéré. 
de celles-ci aux sollicitations d'un nombre important d'agneaux : en 30 jours d'allaitement, les brebis allaitant 3 agneaux produisent en moyenne 80 litres de lait contre 49,5 pour les brebis allaitant un seul agneau né de portées de 2 ou 3 agneaux, soit une supériorité de $62 \mathrm{p}$. Ioo. Ce premier travail réalisé avec un nombre très réduit d'animaux a été poursuivi par de nouvelles observations sur les brebis de race pure Romanov en allaitement d'une part et sur les brebis croisées Romanov-Berrichon allaitant 2 agneaux d'autre part. Ces nouveaux résu'tats ont déjà été inclus partiellement et avant analyse statistique dans les articles de synthèse réalisés par THERIEZ et TISSIER (I975) et RICORDEAU et al., (I978) sur les travaux effectués par 1'I.N.R.A. avec la race Romanov.

\section{II. - Matériel et méthodes}

L'évaluation des aptitudes laitières de brebis de race pure ou croisées Romanov, est effectuée en faisant appel à 2 types de comparaisons :

- Production laitière de brebis Romanov de race pure allaitant I ou 3 agneaux nés de naissances multiples (portées de 2, 3 ou 4 agneaux).

- Production laitière de brebis croisées Romanov ayant mis bas et allaitant 2 agneaux.

\section{I. - Variation de la production laitière de brebis de race pure Romanov selon le nombre d'agneaux allaités (I ou 3). Comparaison $I\left({ }^{*}\right)$}

Au cours des 3 campagnes I970, I972 et I973, des brebis de race pure Romxnov, âgées de $\mathrm{I}$ à 7 ans et ayant réalisé des naissances " multiples " (au moins 2 agneaux), sont réparties entre 6 et $I 2 \mathrm{~h}$ après l'agnelage ( 3 à 5 jours en $\mathrm{I} 970$ ) selon le nombre d'agneaux allaités : I ou 3 agneaux (tabl. 2). Les brebis ayant eu une naissance gémellaire sont automatiquement affectées au lot " I agneau allaité " tandis que pour les naissances triples ou quadruples il est possible de choisir entre $\mathrm{I}$ agneau ou 3 agneaux allaités. Les agneaux surnuméraires (au-delà de I ou de 3 selon le lot) sont séparés de leur mère au moment de la constitution des lots et mis en allaitement artificiel (tab1. 3). Le lait reconstitué est distribué ad libitum à la température ambiante de la bergerie $\left(\mathrm{I} 5^{\circ}\right)$. Pour tous les agneaux (sous la mère ou allaitement artificiel) le sevrage intervient à 42 jours selon le procédé utilisé couramment dans les élevages laitiers (distribution de foin et d'aliment concentré à partir de la $3^{\mathrm{e}}$ semaine). Le fonctionnement détaillé de cette unité d'allaitement artificiel a été décrit par PIESTRA k et al. (I975). Nous n'avons retenu pour l'interprétation des résultats que les brebis dont le nombre d'agneaux allaités n'a pas varié depuis la constitution des lots jusqu'au sevrage : sur l'ensemble des 3 années de comparaison les résultats interprétables concernent 86 agneaux sev rés et 35 misesbas ( $2 \mathrm{I}$ brebis) sur un total de I02 agneaux provenant de 40 mises-bas de 2 agneaux et plus.

(*) Travail effectué au Domaine expérimental I.N.R.A. de $\mathrm{I}_{\mathbf{1}}$ a Fage ( 23350 Roquefort) sous la responsabilité de B. MrRman et P. Daury. 
TABI,EAU 2

Répartition des brebis selon le nombre d'agneaux allaités, la campagne et l'âge. Comparaison I Number of ewes with $\mathrm{x}$ or 3 lambs in suckling (I), by year (2) and by age (3). Experiment I

\begin{tabular}{|c|c|c|c|}
\hline \multirow[b]{2}{*}{ Campagne (2) } & \multirow[b]{2}{*}{ Age des mères (3) } & \multicolumn{2}{|c|}{$\begin{array}{l}\text { Nombre d'agneaux } \\
\text { allaités (I) }\end{array}$} \\
\hline & & I & 3 \\
\hline $1970 . .$. & 4 ans & 5 & 3 \\
\hline 1972... & $\begin{array}{c}6 \text { ans } \\
2 \text { ans } \\
\text { I an } \\
\text { Tous âges }\end{array}$ & $\begin{array}{r}0 \\
7 \\
3 \\
10\end{array}$ & $\begin{array}{l}2 \\
0 \\
3 \\
5\end{array}$ \\
\hline $1973 .$. & $\begin{array}{c}7 \text { ans } \\
3 \text { ans } \\
2 \text { ans } \\
\text { Tous âges }\end{array}$ & $\begin{array}{l}0 \\
3 \\
3 \\
6\end{array}$ & $\begin{array}{l}2 \\
2 \\
2 \\
6\end{array}$ \\
\hline Total & ...... . & $2 \mathrm{I}$ & I4 \\
\hline
\end{tabular}

2.2. - Niveau de production laitière de brebis croisées Romanov allaitant 2 agneaux. Comparaison II (*)

La production laitière de brebis croisées Romanov $\times$ Berrichonne $d u$ Cher allaitant 2 agneaux a été contrôlée au cours de l'année r975. Les brebis appartiennent à 4 types génétiques dont la création et les conditions d'élevage ont été décrites par RICORDEAU et al. (I976b) : FI (Romanov $\times$ Berrichonne $d u$ Cher), F2 (FI $\times$ FI), CR Romanov (Romanov $\times$ FI), CR Berrichonne du Cher (Berrichonne du Cher $\times \mathrm{FI}$ ). Ces brebis ont été saillies au cours de 1'été I974 par les béliers de race Berrichonne $d u$ Cher (RICORDEAU et al., I976a) et ont effectué leur $4^{\mathrm{e}}$ mise-bas du I3 janvier au 4 mars I 975 . Seules ont été retenues pour les présentes observations les brebis ayant agnelé de façon relativement groupée du $\mathrm{x} 7$ janvier au I7 février et ayant mis-bas et élevé 2 agneaux. Durant toute la durée de l'expérience, un même régime alimentaire, à base de foin et d'aliment concentré est distribué aux brebis des différents génotypes. Les agneaux ont accès à volonté à un distributeur d'aliment concentré (alimentation ad libitum) dès la $2^{\mathrm{e}}$ semaine.

(*) Travail effectué au Domaine expérimental I.N.R.A. de Langlade sous la responsabilité de F. EYCHENNE. 


\section{TABLEAU 3}

Répartition des agneaux vivants à 30 jours par campagne et par lot expérimental selon la taille de portée à la naissance (mode de naissance) .Comparaison I

Number of the lambs living at 30 days in reference to the group (I): I or 3 lambs with the mother or artificial rearing; the year (2) and the type of lambing (3). Experiment I.

\begin{tabular}{|c|c|c|c|c|c|}
\hline \multirow{2}{*}{ Lot d'élevage (I) } & \multirow{2}{*}{ Campagne (2) } & \multicolumn{4}{|c|}{ Mode de naissance (3) } \\
\hline & & Double & Triple & Quadruple & Tous modes \\
\hline $\begin{array}{c}\text { I agneau } \\
\text { sous la mère }\end{array}$ & $\begin{array}{l}1970 \\
1972 \\
1973 \\
3 \text { années }\end{array}$ & $\begin{array}{r}1 \\
9 \\
5 \\
15\end{array}$ & $\begin{array}{l}4 \\
\mathrm{I} \\
\mathrm{I} \\
6\end{array}$ & $\begin{array}{l}- \\
-\end{array}$ & $\begin{array}{r}5 \\
10 \\
6 \\
21\end{array}$ \\
\hline $\begin{array}{l}3 \text { agneaux } \\
\text { sous la mère }\end{array}$ & $\begin{array}{l}197^{\circ} \\
197^{2} \\
1973 \\
3 \text { années }\end{array}$ & $\frac{-}{-}$ & $\begin{array}{r}6 \\
15 \\
15 \\
36\end{array}$ & $\frac{3}{3}$ & $\begin{array}{r}9 \\
15 \\
18 \\
42\end{array}$ \\
\hline $\begin{array}{l}\text { Allaitement } \\
\text { artificiel }\end{array}$ & $\begin{array}{l}1970 \\
1972 \\
1973 \\
3 \text { années }\end{array}$ & $\begin{array}{r}1 \\
8 \\
5 \\
14\end{array}$ & $\begin{array}{l}5 \\
1 \\
1 \\
7\end{array}$ & $\frac{\mathrm{I}}{\mathrm{I}}$ & $\begin{array}{r}7 \\
9 \\
7 \\
23\end{array}$ \\
\hline Tous lots & $\begin{array}{l}1970 \\
197^{2} \\
1973 \\
3 \text { années }\end{array}$ & $\begin{array}{r}2 \\
17 \\
10 \\
29\end{array}$ & $\begin{array}{l}15 \\
17 \\
17 \\
49\end{array}$ & $\frac{4}{4}$ & $\begin{array}{l}21 \\
34 \\
31 \\
86\end{array}$ \\
\hline
\end{tabular}

\section{3. - Enregistrements}

Dans les 2 Comparaisons (I et II) la production laitière des brebis est estimée à intervalles de 7 jours à l'aide de la " méthode hormonale ": deux vidanges de la mamelle sont réalisées à $4 \mathrm{~h}$ d'intervalle ( $\mathrm{Ir} \mathrm{h}$ et $\mathrm{I} 5 \mathrm{~h}$ environ) par injection intrajugulaire de 5 UI d'ocytocine en 3 doses successives (2UI - 2 UI - IUI) effectuées à $2 \mathrm{mn}$ d'intervalle et suivies d'une traite manuelle. La quantité de lait recueillie au cours de la $2^{\mathrm{e}}$ vidange est multipliée par 6 pour fournir une estimation de la quantité de lait secrétée en $24 \mathrm{~h}$. Les premières observations sont effectuées 3 à 5 jours après la mise-bas et la dernière 38 à 40 jours. On a estimé le lait total produit en 42 jours, par la méthode FLEICHMANN en extrapolant jusqu'au jour de mise-bas la production enregistrée lors du premier contrôle et jusqu'au $4^{2}$ jour la production au dernier contrôle.

Les brebis sont pesées à la mise-bas. Dans la Comparaison I le poids atteint 42 jours après la mise-bas (sevrage) est estimé par intrapolation à partir de pesées effectuées dans le troupeau tous les 2 I jours. Dans la Comparaison II ce poids 
est directement mesuré. Les agneaux sont également pesés tous les 2 I jours dans la Comparaison I tandis que la pesée est hebdomadaire dans la Comparaison II. L'indice de consommation a été défini comme la quantité de lait (en litres) nécessaire pour obtenir I $\mathrm{kg}$ de croît.

\section{III. - Résultats}

\section{I. - Brebis Romanov allaitant I ou 3 agneaux (Comparaison I).}

Sur les ro2 agneaux d'origine provenant de 40 portées de 2 agneaux et plus, la mortalité périnatale est de 3,9 p. Ioo (4 agneaux morts). Parmi les agneaux utilisés dans l'expérience on enregistre également 4 pertes avant I mois (4 p. roo)

\section{TABLEAU 4}

Performances zootechniques et production laitière durant les 30 premiers jours d'allaitement (méthode hormonale) selon le nombre d'agneaux sous la mève. Comparaison I

Entre paranthèses : performances " 3 agneaux allaités " exprimés en p. xoo des performances "I agneau allaité "

Live weight at lambing (1), milk yield during the first 30 days (2), total growth:(3) and consumption indice (milk yield/total growth) (4), relative to the number of lambs reared In parenthesis " 3 lambs " performances by report to the "I lamb" ones p. Ioo). Fxperiment I

\begin{tabular}{|c|c|c|c|c|c|c|}
\hline Année & \begin{tabular}{|c|} 
I,ot \\
expéri- \\
mental \\
(nombre \\
d'agneaux \\
sous la mère)
\end{tabular} & Effectif & $\begin{array}{l}\text { Poids } \\
\text { à la } \\
\text { mise-bas } \\
\text { (kg) } \\
\text { (I) }\end{array}$ & $\begin{array}{l}\text { Quantité } \\
\text { de lait } \\
\text { o-3o jours } \\
\text { (1) } \\
\text { (2) }\end{array}$ & $\begin{array}{c}\text { Croissance } \\
\text { de la } \\
\text { portée } \\
\text { o-3o jours } \\
\text { (kg) } \\
(3)\end{array}$ & $\begin{array}{c}\text { Quantité } \\
\text { de lait / } \\
\text { Croissance } \\
\text { o-3o jours } \\
(4)\end{array}$ \\
\hline $1970 .$. & $\begin{array}{l}\text { I } \\
3\end{array}$ & $\begin{array}{l}5 \\
3\end{array}$ & $\begin{array}{l}6 \mathrm{I}, 8 \\
56,7\end{array}$ & $\begin{array}{l}49,5 \\
80,2(\mathrm{x} 62, \mathrm{o})\end{array}$ & $\begin{array}{r}6,8 \\
16,9(248,7)\end{array}$ & $\begin{array}{l}7,3 \\
4,7\end{array}$ \\
\hline $1972 .$. & $\begin{array}{l}\text { I } \\
3\end{array}$ & $\begin{array}{r}10 \\
5\end{array}$ & $\begin{array}{l}39,3 \\
47,4\end{array}$ & $\begin{array}{l}3 \mathrm{I}, 3 \\
67,0 \quad(2 \mathrm{I} 4, \mathrm{I})\end{array}$ & $\begin{aligned} 5,8 & \\
\mathrm{I} 4, \mathrm{I} & (243, \mathrm{I})\end{aligned}$ & $\begin{array}{l}5,4 \\
4,7\end{array}$ \\
\hline 1973. & $\begin{array}{l}\text { I } \\
3\end{array}$ & $\begin{array}{l}6 \\
6\end{array}$ & $\begin{array}{l}45,3 \\
52,3\end{array}$ & $\begin{array}{l}33,7 \\
59,4 \quad(\text { I } 76,2)\end{array}$ & $\begin{array}{r}7,4 \\
\mathrm{I} I, 7 \quad(\mathrm{I} 55,4)\end{array}$ & $\begin{array}{l}4,6 \\
5,1\end{array}$ \\
\hline 3 années $(*)$ & $\begin{array}{l}\text { I } \\
3\end{array}$ & $\begin{array}{l}2 \text { I } \\
\text { I } 4\end{array}$ & $\begin{array}{l}48,8 \\
5^{2,}, \mathrm{I}\end{array}$ & $\begin{array}{l}3^{8,2} \\
68,9(180,4)\end{array}$ & $\begin{array}{r}6,7 \\
\text { I } 4,2 \quad(2 \text { I I }, 8)\end{array}$ & $\begin{array}{l}5,7 \\
4,9\end{array}$ \\
\hline $\begin{array}{r}3 \text { années } \\
(* *)\end{array}$ & $\begin{array}{l}\text { I } \\
3\end{array}$ & $\begin{array}{l}2 \mathrm{I} \\
\mathrm{I} 4\end{array}$ & $\begin{array}{l}48,8 \\
52, \mathrm{I}\end{array}$ & $\begin{array}{l}37,8 \\
65,3 \quad(\text { I } 72,7)\end{array}$ & $\begin{array}{r}6,6 \\
\text { I } 3,7(207,6)\end{array}$ & $\begin{array}{l}5,7 \\
4,8\end{array}$ \\
\hline
\end{tabular}

(*) Estimées "moindre carrés ". Analyse de variance sans covariable.

(**) Fistimées " moindre carrés ". Analyse de variance avec covariable Poids à la mise-bas. 
se répartissant pour moitié entre les lots élevés triples (4,3 p. I0o) et simples $(8,3 \mathrm{p}$. IOo). Aucune mortalité n'est enregistrée parmi les agneaux en allaitement artificiel.

Des différences importantes sont observées entre années pour le poids à la mise-bas des brebis $(\mathrm{P}<0, \mathrm{oI}$; tabl. 4) et dans une moindre mesure pour le niveau moyen de production laitière et la croissance de la portée. Cela tient vraisemblablement à 1'âge moyen des animaux qui est beaucoup plus faible en 1972 (brebis de $\mathrm{I}$ an et 2 ans essentiellement) et en I973 (brebis de 2 et 3 ans) qu'en I 970 (brebis de 4 ans). Les brebis allaitant 3 agneaux ont un poids à la mise-bas légèrement plus important $\left(5^{2, I}\right.$ vs. $48,8 \mathrm{~kg}$ pour les brebis allaitant I agneau). Il est donc possible que la différence de production laitière entre les brebis allaitant I et 3 agneaux soit surestimée par cette légère différence. On observe effectivement que l'écart de production entre les 2 catégories de brebis est atténué lorsque l'on tient compte de ce poids comme covariable dans l'analyse de variance. Les mêmes observations peuvent être faites sur la croissance de la portée entre o et 30 jours (tab1. 4).

La production laitière de o à 30 jours des brebis allaitant 3 agneaux et $\mathbf{I}$ agneau est respectivement de 65,3 et 37,81 pour l'ensemble des 3 années d'observations et après correction pour le poids à la mise-bas (tabl. 4) : ceci correspond pour les portées triples par rapport aux portées simples à une quantité de lait consommée supérieure de $73 \mathrm{p}$. roo. La croissance totale de la portée étant accrue dans des proportions encore plus importantes ( 108 p. roo), il en résulte que l'indice de consommation des agneaux élevés triples $(4,8)$ est meilleur que celui des agneaux élevés simples $(5,8)$ pour la période $0-30$ jours considérée. L'écart maximum de

\section{TABLEAU 5}

Analyses de variance et résultats des tests de signification statistique pour le poids à la mise-bas, la quantité de lait o-3o jours et la croissance de la portée durant la même période. Comparaison I

Elements and results of the analysis of variance upon the lambing weight (I)

and the milk yield o-3o days (2) of the ewes, and the total growth 0 -3o days of the lambs (3). Experiment I

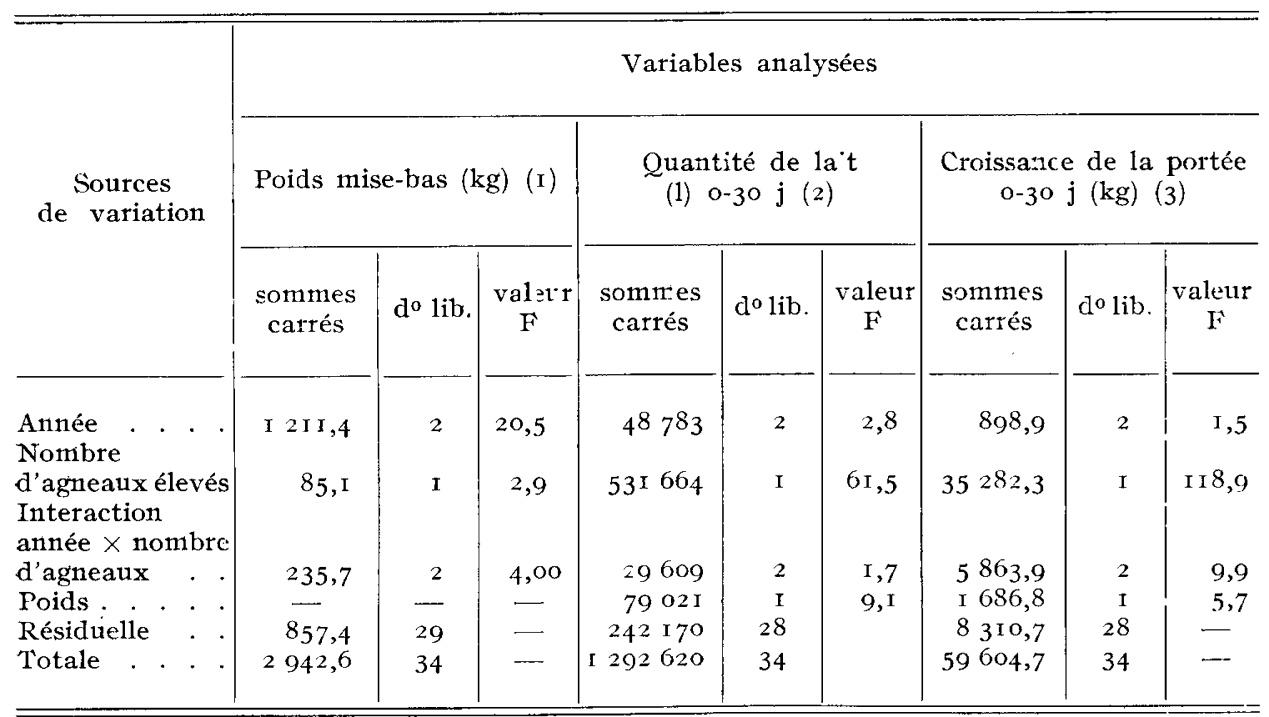



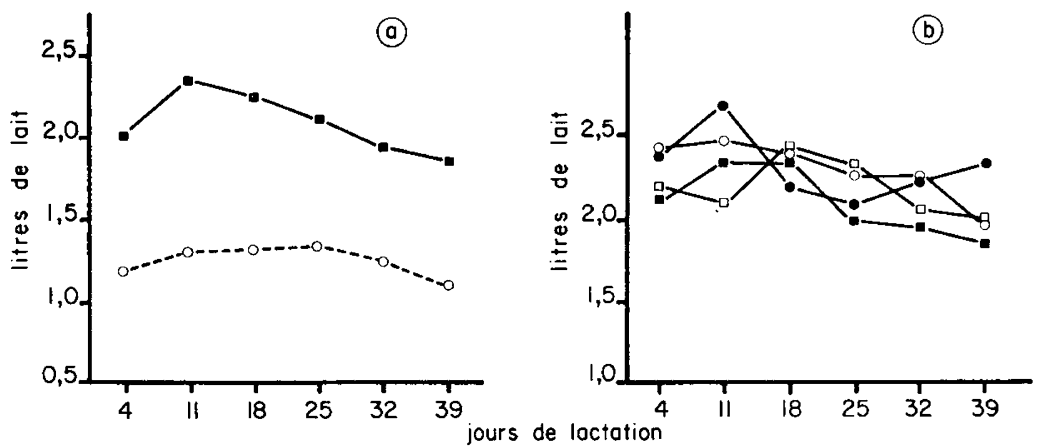

FIG. I. - Évolution de la production laitière des brebis.

a) Brebis Romanov allaitant $\mathrm{I}$ ou 3 agneaux $\left(\mathrm{P}_{1}\right.$ et $\left.\mathrm{P}_{3}\right)$.

(Moyennes estimées après correction pour l'effet de l'année et du poids à la mise-bas).

Comparaison I O - ... O I agneau sous la mère

Jour II $P_{3} / P_{1}$ p. Ioo $=179,6$

p. $100=179,6$
p. $100=159,7$

b) Brebis de différents génotypes croisés

Romanov $\times$ Berrichonne du Cher allaitant 2 agneaux

Comparaison $I I \bigcirc 3 / 4$ Berrichon

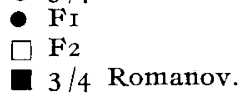

Evolution of milk yield during the first 6 weeks of the lactation.

a) Romanov ewes suckling $\mathrm{I}$ or 3 lambs (Means squares estimated corrected for the year and lambing weight effects). Experiment $I$.

b) Crossed Romanov ewes with 2 lambs in suckling. Experiment $I I$.

\section{TABLEAU 6}

Performances de croissance des agneaux selon le mode d'allaitement : I ou 3 agneaux allaités ou allaitement artificiel (estimations obtenues par la méthode des moindres carrés). Comparaison I
(a) $n=20$
(b) $n=3^{8}$
(c) $n=22$

Weight at different ages (1). Growth rate (2) of individual lambs in respect to the type of rearing ( $I$ or 3 lambs with the mother, artificial rearing). Experiment I

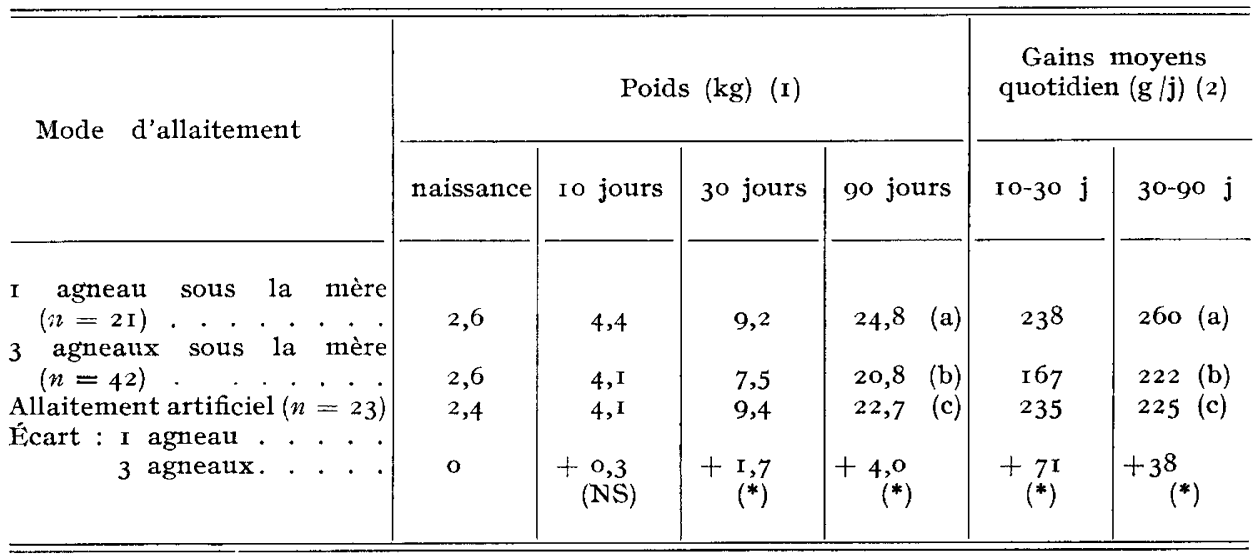


production entre les 2 lots de brebis est atteint au cours de la $2^{\mathrm{e}}$ semaine de lactation où la production des brebis allaitant 3 agneaux est supérieure de 80 p. Ioo à celle des brebis avec un seul agneau (fig. $\mathrm{r} a$ ), tandis qu'elle n'est plus que de 60 p. Ioo à 39 jours, à la veille du sevrage.

La vitesse de croissance individuelle des agneaux élevés triples est inférieure de $30 \mathrm{p}$. Ioo à celle des agneaux élevés simples ( $167 \mathrm{~g} / \mathrm{j}$ vs $238 \mathrm{~g} / \mathrm{j}$, tabl. 6), alors que les agneaux allaités artificiellement obtiennent la même croissance que les agneaux simples $(235 \mathrm{~g} / \mathrm{j})$. Après le sevrage l'écart entre simples et triples se réduit avec la réalisation d'une croissance compensatrice par les agneaux triples dont le poids moyen ne rejoint cependant pas celui des agneaux simples. La prise en compte des croissance obtenues en phase d'allaitement permet d'obtenir une nouvelle estimée de la production laitière des brebis, méthode dont l'efficacité a été testée notamment par POUJARDIEU (I969). Nous avons utilisé ici les équations de régression établies par RICORDEAU et BOCCARD (I96I) pour des agneaux simples $\lambda_{1}(\mathrm{Y}=4,59 \mathrm{X}+3 \mathrm{II})$ et doubles $\lambda_{2}(\mathrm{Y}=3,50 \mathrm{X}+68 \mathrm{o})$ de race Préalpes $d u$ Sud, contrôlés entre o et 30 jours (tab1. $7 a$ ). Les estimées obtenues par ce calcul sont très semblables à celles qui ont été mesurées par la méthode hormonale.

\section{2. - Brebis croisées allaitant 2 agneaux (Comparaison II)}

On observe une tendance à la diminution de la production laitière lorsque la proportion de sang Romanov augmente (tabl. 8, fig. $\mathrm{I} b$ ). C'est ainsi que le niveau de production $0-42$ jours des brebis $3 / 4$ Romanov est inférieur de $7,7 \mathrm{p}$. Ioo à celui des I/4 Romanov. Ces mêmes brebis $3 / 4$ Romanov sont d'ailleurs les plus légères à la mise-bas et au sevrage, et présentent la perte de poids minimum entre la mise-bas et le sevrage. L'introduction d'une covariable " poids à la mise-bas" dans l'analyse de variance a pour conséquence de réduire les différences entre génotypes.

Des différences plus notables entre types génétiques des mères se manifestent pour la vitesse de croissance des agneaux (bien que les différences observées ne soient toujours pas significatives, tabl. 9) : les brebis Fr confèrent à leurs produits

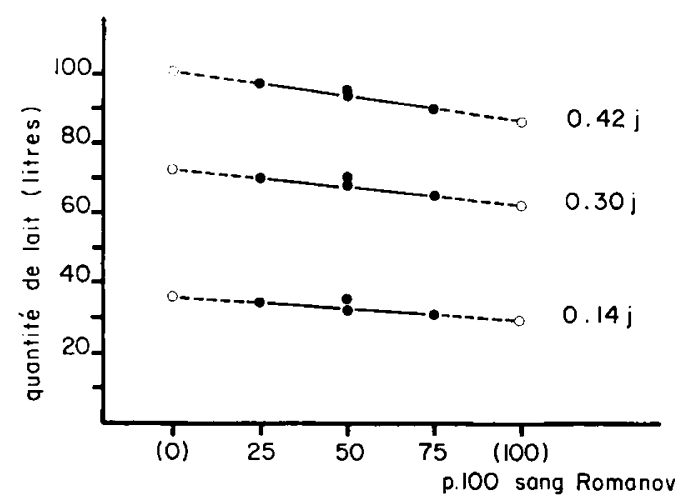

FIG 2. - Production laitière moyenne des brebis appartenant aux génotypes croisés Romanov $\times$ Berrichonne du Cher, allaitant 2 agneaux, selon la période de lactation considéríe (•). Extrapolation pour des brebis de races pures Romanov et Berrichonne du Cher (O). Comparaison II. Mean milk yield of crossed Romanov ewes with a lambs in suckling, at different steps of the lactation during the firts 6 weeks. 


\section{TABLEAU 7}

Croissances moyennes journalières des agneaux de o à 30 jours et estimation de leur consommation de lait

(méthode hormonale et estimation par régression, en considérant une densité moyenne $d=I, 040$ )

Mean growth rate of individual lambs o-3o days (1)

and estimation of milk yield by hormonal method (2) or by regression method (3)

(Mean used density $d=I, 040$ ). Experiments $I(a)$ and $I I(b)$

a) Comparaison $I$

\begin{tabular}{|c|c|c|c|}
\hline \multirow{2}{*}{$\begin{array}{l}\text { Isot expérimental } \\
\text { (nombre d'agneaux sous la mère) }\end{array}$} & \multirow{2}{*}{$\begin{array}{c}\text { Croissance } \\
\text { moyenne } \\
\text { journalière } \\
\text { individuelle }(\mathrm{g} / \mathrm{j}) \\
(\mathrm{I})\end{array}$} & \multicolumn{2}{|c|}{$\begin{array}{l}\text { Quantité de lait } \\
\text { consommé par agneau (g) }\end{array}$} \\
\hline & & $\begin{array}{l}\text { Métliode } \\
\text { hormonale (2) }\end{array}$ & Régression (3) \\
\hline $\mathbf{I}$ & 220 & I 3 IO & I 323 \\
\hline 3 & 152 & 753 & 759 \\
\hline
\end{tabular}

b) Comparaison $I I$

\begin{tabular}{|c|c|c|c|}
\hline \multirow{2}{*}{$\begin{array}{l}\text { Lot expérimental } \\
\text { (génotype) }\end{array}$} & \multirow{2}{*}{\begin{tabular}{|c|} 
Croissance \\
moyenne \\
journalières \\
individuelle $(\mathrm{g} / \mathrm{j})$ \\
$(\mathrm{I})$
\end{tabular}} & \multicolumn{2}{|c|}{$\begin{array}{l}\text { Quantité de lait } \\
\text { consommé par agneau (g) }\end{array}$} \\
\hline & & $\begin{array}{l}\text { Méthode } \\
\text { hormonale (2) }\end{array}$ & Régression (3) \\
\hline $\begin{array}{l}\text { C. R. Berrichonne Cher } \\
\text { FI } \\
\mathrm{F}_{2} \\
\text { C. R. Romanov }\end{array}$ & $\begin{array}{l}\text { I } 84 \\
205 \\
\text { I } 79 \\
\text { I } 74\end{array}$ & $\begin{array}{lll}\text { I } & 2 & \text { I } \\
\text { I } & 2 & \text { I } 4 \\
\text { I } & \text { I } 75 \\
\text { I } & \text { I I } 4\end{array}$ & $\begin{array}{r}984 \\
\text { I } 057 \\
966 \\
949\end{array}$ \\
\hline
\end{tabular}

\section{TABLEAU 8}

Performances laitières et variations de poids des mèves selon le génotype. Comparaison II (différences no: significatives, $P>0,05$ )

Milk yield and livs wcight of the ewes at different steps of the lactation for several crossed Romanov gcnotypes. Experiment II

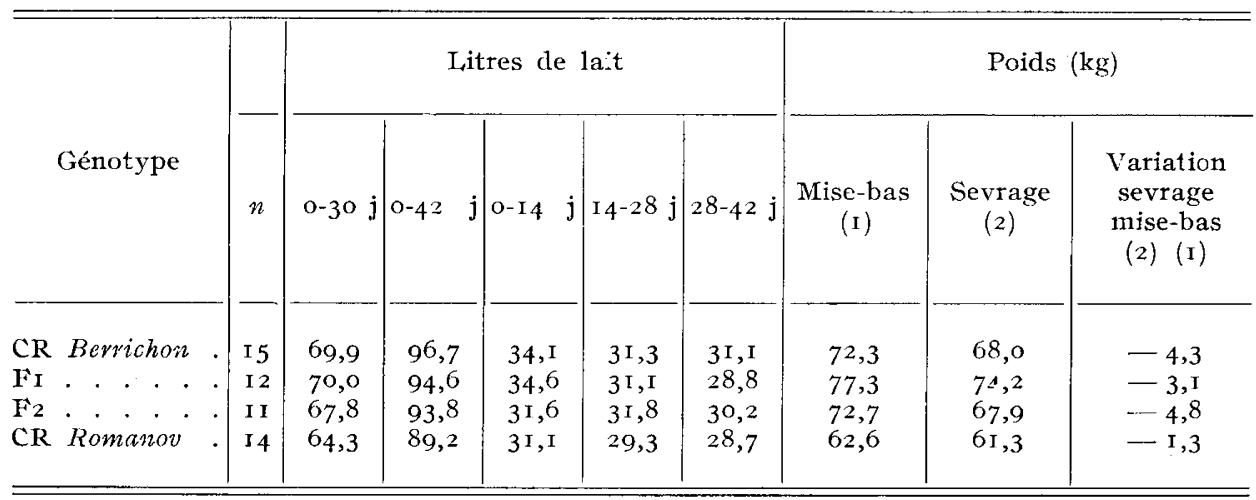


la meilleure vitesse de croissance et les poids les plus élevés à tout âge, les moins bonnes performances étant obtenues avec les brebis 3/4 Romanov. De même, les agneaux issus de femelles $F_{I}$ réalisent les meilleurs indices de consommation.

Au cours des années antérieures (I972, I973 et I974) et dans le même troupeau en $\mathrm{r}^{\mathrm{re}}, 2^{\mathrm{e}}$ et $3^{\mathrm{e}}$ lactation, BoNAITI et HARRACA (I975) ont enregistré également sur les agneaux nés et élevés doubles, des vitesses moyennes de croissance journalières entre ro et 30 jours d'autant plus faibles que les brebis possèdent un pourcentage de sang Romanov important (tabl. ro). Cependant, dans le cas des meilleures

\section{TABLEAU 9}

Croissances moycnnes de la portée et indices de consommation. Comparaison II (différences non significatives)

Mean growth rate of the lambs (I) and corresponding indices of consumption (2) at different steps of the lactation for several crossed Romanov genotypes. Experiment II

\begin{tabular}{|c|c|c|c|c|c|c|c|c|c|c|}
\hline \multirow{2}{*}{$\begin{array}{l}\text { Génotype } \\
\text { des mères }\end{array}$} & \multirow{2}{*}{$\begin{array}{l}\text { Croissance } \\
\text { moyenne } \\
\text { journalière } \\
0-30 \mathrm{j}(\mathrm{g})\end{array}$} & \multicolumn{4}{|c|}{ Gains moyens $(\mathrm{kg})(\mathrm{I})$} & \multicolumn{5}{|c|}{ Indices de consommation (2) } \\
\hline & & O-I $4 \mathrm{j}$ & $14-28 j$ & $28-42 j$ & $0-42 j$ & $\mid 0-30 \mathrm{j}$ & O-I $4 \mathrm{j}$ & $14-28 j$ & $28-42 j$ & $0-42 j$ \\
\hline CR Berrichonne. & 369 & 4,8 & 5,3 & 7,3 & I 7,7 & 6,3 & 7,2 & 5,9 & 4,4 & 5,5 \\
\hline FI . . . . . . & 410 & 5,6 & 5,8 & 8,4 & I9,8 & 5,7 & 6,3 & 5,4 & 3,6 & 4,8 \\
\hline $\mathrm{F}_{2} \cdot . \cdot . \cdot$. & 357 & 4,5 & 5,3 & 6,3 & 16,2 & 6,8 & 7,4 & 6,4 & 5,6 & 6,3 \\
\hline CR Romanov. . & 349 & 4,7 & 5,2 & $7, \mathbf{I}$ & 16,9 & $6, \mathrm{I}$ & 6,9 & 5,7 & 4,2 & 5,3 \\
\hline
\end{tabular}

TABLEAU IO

Vitesses moyennes de croissances journalières entre Io et 30 jours $(g / j)$ d'agneaux nés doubles sclon le génotype des mères et le mode d'élevage au cours des 3 premières mises-bas.

Comparaison II. (BonaITI et HARRACA, I975)

Mean growth rate of individual twin lambs reared as twin, single or artificially (I) and born from crossed Romanov ewes (2) during their three first lambings. Experiment II

\begin{tabular}{|c|c|c|c|c|c|c|c|c|c|}
\hline \multirow{2}{*}{$\begin{array}{l}\text { Campa- } \\
\text { gne }\end{array}$} & \multirow[b]{2}{*}{ Mode d'Élevage (I) } & \multicolumn{2}{|c|}{ CR Berrichon } & \multicolumn{2}{|c|}{$F_{I}$} & \multicolumn{2}{|c|}{$\mathrm{F}_{2}$} & \multicolumn{2}{|c|}{ CR Romanov } \\
\hline & & $n$ & $\bar{x}$ & $n$ & $\bar{x}$ & $n$ & $\bar{x}$ & $n$ & $\bar{x}$ \\
\hline I972 & Allaitement artificiel . . . & 3 & 294 & 29 & 268 & 26 & 285 & 30 & 285 \\
\hline I973 & 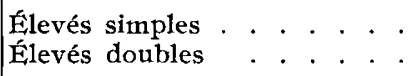 & 3 & 262 & $\begin{array}{l}10 \\
27\end{array}$ & $\begin{array}{l}278 \\
243\end{array}$ & $\begin{array}{r}9 \\
19\end{array}$ & $\begin{array}{l}260 \\
23 I\end{array}$ & $\begin{array}{l}12 \\
25\end{array}$ & $\begin{array}{l}288 \\
203\end{array}$ \\
\hline I 974 & Élevés doubles . . . . . . & 32 & 230 & 27 & 220 & 24 & 218 & IO & 216 \\
\hline
\end{tabular}


performances sur les agneaux nés doubles mais élevés simples ou artificiellement sont observées avec les mères $3 / 4$ Romanov. Ce fait incite à penser que le facteur limitant principal n'est pas le potentiel de croissance des agneaux mais bien le potentiel laitier des mères.

Les corrélations entre la quantité de lait consommée par les agneaux et leur croît baisse de façon sensible pour tous les génotypes lorsque s'avance la lactation et particulièrement entre 28 et 42 jours (tabl. II). Ceci justifie que 1 'on s'intéresse principalement aux performances enregistrées au cours du premier mois (0-30 jours). On observe corrélativement une diminution de l'indice de consommation durant la même période (tabl. 9) ce qui pourrait être dû̀ à l'intervention d'une part de plus en plus importante d'aliments concentrés dans la ration à partir de la $2^{\text {e }}$ quinzaine.

\section{TABLEAU I I}

Corrélation entre variables de la production laitiève et de crô̂t des agneaux aux diffévents âges. Comparaison II

Correlation between milk yield and growth rate of the lambs during the first 6 weeks of the lactation. Experiment II

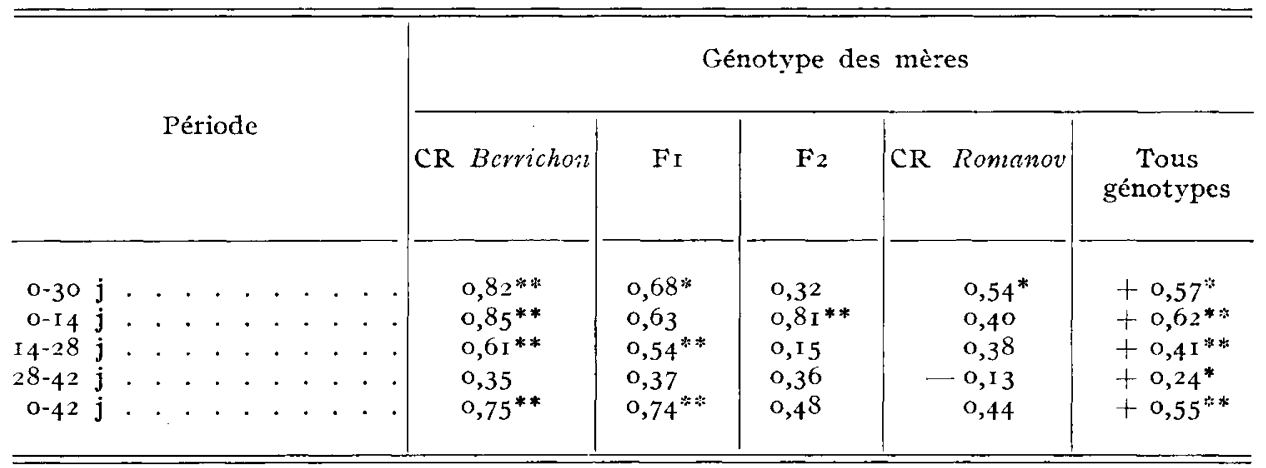

On peut constater que contrairement aux résultats de la Comparaison $\mathrm{I}$, les estimations de production laitière obtenues par régression à partir des croissances observées sont toujours inférieures ( 160 à $230 \mathrm{~g}$ ) à celles de la méthode hormonale (tabl. $7 b$ ). Cependant le classement des génotypes n'est pas modifié.

\section{Discussion}

Les observations relatives à des agneaux triples sont très rares dans la littérature et ne portent en tout cas que sur un nombre restreint d'animaux. Il est vrai que ces travaux concernent toujours des races pour lesquelles la naissance de 2 ou 3 agneaux est peu fréquente sinon même exceptionnelle. Da plus il s'agit généralement d'observations faites à l'occasion d'expérimentations ayant des préoccupations principales d'ordre physiologique (fonctionnement de la mamelle et sécrétion du lait) ou nutritionnel (besoins supplémentaires d'énergie pour les 
brebis nourrissant plus d'un agneau, efficacité alimentaire des agneaux élevés multiples. Or la race Romanov, dont les brebis donnent naissance couramment à $2,3,4$ agneaux, constitue un matériel animal particulièrement intéressant pour l'étude des interactions mère $\times$ produits. Celles-ci peuvent être envisagées entre la vigueur de 1'agneau et la quantité de lait disponible de la mère comme BRUNEL et al. (I975) et FLAMANT, CASU (I977) 1'ont déjà suggéré.

I. La viabilité de 1'agneau peut être considérée comme un critère maternel liée à l'aptitude des mères à donner suffisamment de lait pour faire vivre les jeunes. Le seuil au-dessous duquel ce caractère peut se manifester n'a pas été atteint dans nos comparaisons : les faibles effectifs concernés, tout comme les conditions particulières d'élevage liées à la réalisation des expériences (notamment une surveillance plus grande des animaux expérimentaux que dans un troupeau normal) ne permettent pas de faire intervenir valablement ici le critère "viabilité " comme critère d'évaluation des catégories de brebis concernées (nombre d'agneaux allaités ou types génétiques). Il n'apparaît pas par exemple que la viabilité des agneaux nés de portées triples ou quadruples ait été plus faible, ce qui aurait pu avoir pour effet de biaiser les comparaisons portant ensuite sur la production laitière. Ceci n'est évidemment pas toujours le cas et les résultats de RICORDEAU et al. (I977) et FLAMANT et al. (I977) portant sur des effectifs beaucoup plus importants et dans des conditions d'élevage moins " surveillées ", permettent de se faire une opinion plus complète sur la viabilité des agneaux Romanov issus de portées multiples. Celle-ci bien qu'inférieure à celle des agneaux nés simples, se situe à un niveau supérieur à celui des agneaux d'autres races à taille de portée égale.

2. En ce qui concerne les quantités de lait mesurées sur l'ensemble des 3 années considérées, la supériorité des brebis allaitant 3 agneaux est sensiblement du même ordre de grandeur que lors des essais préliminaires de I970 (Flamant et LABUSSIÈRE, I972) : 72,7 p. IOO vs. $62,0 \mathrm{p}$. I00. Ces valeurs sont également très proches de celles obtenues par KaLAISSA KIs et al. (I977) avec des brebis Chios, Frisonnes ou croisées Frisonne-Chios élevant 3 agneaux, par rapport aux brebis élevant un seul agneau (62 à 92 p. Ioo selon les lots). Mais il est intéressant de noter que les écarts sont beaucoup plus importants lors du maximum de la lactation : 80 p. Ioo dans notre cas (fig. I); 7I à I05 p. IOO pour KALAIssakis et al. (I977), soit environ ro points de plus que pour la production totale au cours des. 6 ou 8 premières semaines de lactation. PEART et al. (I972) constatent également un pic de production très élevé pour des brebis élevant 3 et 4 agneaux par rapport aux brebis avec un seul agneau, la quantité de lait étant supérieure de $9 \mathrm{r}$ p. roo au cours des 4 premières semaines. Les niveaux moyens de production que nous obtenons ici avec 3 agneaux Romanov allaités au cours du premier mois sont en définitive identiques à ceux de MAGomedov (I975a) obtenus sur la même race : $65,3 \mathrm{~kg}$ contre 64 à $68 \mathrm{~kg}$ selon le type des brebis. Ces valeurs sont également très proches de celles observées par ANTILLA (I975) avec des brebis Finnoises (I,8 $\mathrm{kg} / \mathrm{j}$ ). Les besoins alimentaires de ces brebis élevant plusieurs agneaux peuvent donc être accrus dans des proportions très élevées et ceci d'autant plus que la meilleure efficacité alimentaire des agneaux, déjà observée par RICORDEAU et BOCCARD (r97I), GARNER et al. (r964) et PEART (I972), est explicable au moins en partie par une valeur énergétique plus grande du lait sécrété (GARNER et HoGUE, I964).MAGOMEDOV (I975b) observe précisément une élévation de la richesse du lait des brebis Romanov avec le nombre d'agneaux allaités (TB : I0,7II,3-I3,2 $\mathrm{g} / \mathrm{kg}$ de lait respectivement pour 2-3-4 agneaux).

3. La plus grande surface placentaire des brebis portant plusieurs agneaux pourrait être à l'origine de capacités lactées supérieures par suite de sécrétions 
hormonales plus importantes jouant un rôle dans la mammogénèse et la lactogénèse (LABUSSIÈRE, r979). Mais la production laitière plus importante de brebis élevant plusieurs agneaux tient aussi à la vidange plus fréquente de la mamelle (LABUSSIÈRE et CombAUD, I978) qui induit une stimulation plus grande du réflexe d'éjection et permet aux agneaux de tirer largement partie des aptitudes laitières de leurs mères : l'observation courante des éleveurs et des techniciens témoigne de la vivacité des jeunes agneaux Romanov vis-à-vis de leur mère et en général de l'ensemble des brebis du troupeau (existence d'agneaux voleurs). Ceci fait d'ailleurs ressortir les limites de la Comparaison II réalisée avec des brebis croisées appartenant à différents génotypes. On observe en effet dans ce cas le résultat d'une interaction entre la vigueur de l'agneau et 1'aptitude laitière de la mère (FLAmant et CASU, I977). Les valeurs observées pourraient donc être attribuées aussi bien au génotype de l'agneau qu'à celui de sa mère. Pour séparer les 2 effets, il faudrait avoir recours à des dispositifs expérimentaux tels que ceux proposés par MoORE (I966) et qui prévoient dans le cas de portées doubles des échanges d'agneaux entre portées : les brebis Romanov ou croisées Romanov constituent un matériel intéressant pour la mise en œuvre d'expériences de ce type. Cependant nous pouvons admettre de raisonner ici en fonction du type génétique de la mère, qui a une variation plus grande que celui de l'agneau dans notre expérience $(25$ à $75 \mathrm{p}$. Ioo de sang Romanov contre I2,5 à 37,5 p. IOO). On observe alors que l'accroissement du pourcentage de sang Romanov introduit des performances laitières plus faibles. La variation apparaît à peu près linéaire si l'on admet la manifestation d'un léger effet d'hétérosis en FI par rapport aux F2, de sorte que l'extrapolation conduirait à estimer une valeur laitière des brebis Romanov inférieure d'environ 9 p. roo à celle des brebis Berrichonne $d u$ Cher à nombre d'agneaux allaités égal (fig. 2.). Ce raisonnement sur le génotype de la mère apparaît d'ailleurs justifié si nous observons que pour les agneaux doubles élevés simples ou mis en allaitement artificiel (par conséquent sans limitation de consommation lactée) aucune tendance similaire n'apparaît pour la vitesse de croissance, les agneaux ayant le pourcentage de sang Romanov le plus important obtenant une croissance au moins égale aux autres (Bonaitr et Harraca, I975). D'autres résultats comparables existent dans la littérature au sujet des génotypes Finnois. C'est ainsi que CrowLEY et Mc GLOUBHLIN (I972) montrent que la production laitière de brebis croisées Finnoises est supérieure à celle des Finnoises à taille de portée égale. Sommer et al. (I974) obtiennent une plus grande quantité de lait avec les brebis allaitantes Schwarskopf par rapport à des croisées Finnoise $\times$ Schwarzkopf au même niveau d'alimentation, de même que JAKUBEc et al. (r978) avec des brebis Valaques par rapport à des croisées FI Finnoises $\times$ Valaques. Seuls TREACHER et GIBB (I978) comparant les quantités de lait obtenues à la traite avec les brebis croisées Finnoise $\times$ Dorset Horn à celles des brebis Dorset Horn pures n'observent pas de différences.

4. La difficulté d'effectuer des comparaisons en maîtrisant le niveau de stimulation provenant des agneaux conduit à s'intéresser aux quantités de lait recueillies à la traite depuis le début de la lactation afin de se soustraire à l'influence de l'agneau. Les premières observations de FLAMANT et LABUSSIÈRE (I972) tendaient à évaluer les performances laitières des brebis Romanov en dessous de celles des brebis Lacaune avec une traite relativement complète (double pose des gobelets). Dans les conditions de traite simplifiées (une seule pose des gobelets, sans repasse), nous observons (FIAMANT et JACQUIN, I975) que le niveau de production laitière d'un lot de 16 brebis Romanov en $2^{\mathbf{e}}$ lactation à 2 ans s'établit nettement en dessous de celui de 24 brebis Lacaune d'un lot témoin "sans sélection " (66 litres en 122 jours de traite contre r 29 litres en $I 96$ jours de traite depuis la mise-bas). 
La persistance est également moins bonne pour les brebis Romanov: le pourcentage de brebis atteignant $5_{5}$ jours de traite est de 87,5 p. roo pour les Lacaune et seulement $37,5 \mathrm{p}$. Ioo pour les Romanov. Mais dans une telle comparaison on introduit alors un autre paramètre qui peut biaiser les comparaisons entre génotypes, 1'aptitude à la traite. Nous ne disposons actuellement d'aucune observation objective permettant de situer la réponse à la traite des brebis Romanov par rapport aux autres races mieux connues dans ce domaine telles que la Lacaune ou la Sarde.

\section{Conclusion}

I es différentes comparaisons auxquelles nous avons eu recours ont pour objectif une meilleure connaissance des aptitudes laitières des brebis Romanov, pures ou croisées. C'est ainsi que nous avons d'abord évalué l'influence déterminante du nombre d'agneaux allaités sur le niveau de sécrétion de la mamelle. Nous avons tenté ensuite de situer les aptitudes laitières des brebis à niveau de stimulation égal : même nombre d'agneaux allaités ou traite mécanique. Il n'est cependant pas possible d'éliminer totalement l'effet éventuel du génotype de l'agneau dans une comparaison de différents types génétiques de mères, ainsi que 1'incidence de l'aptitude à la traite mécanique surtout si celle-ci est simplifiée.

Dans tous ces essais, il faut noter cependant que les résultats vont toujours dans le sens d'une moindre aptitude laitière apportée par la race Romanov, résultat similaire à celui obtenu par d'autres auteurs avec la race Finnoise. On peut donc se demander s'il ne s'agit pas d'une caractéristique propre aux races très polifiques, à laquelle il faudrait prêter également attention lors des études sur les races $D^{\prime}$ man, Black-Belly, etc...

Admettant la conclusion d'une moindre production laitière des mères, il faut s'interroger maintenant sur les conséquences zootechniques de ce fait pour la production d'agneaux. En race pure ou en croisement de première génération (agneaux FI), ce handicap semble compensé par une meilleure vitalité des agneaux. En revanche la vigueur des agneaux issus d'un croisement terminal de $2^{\circ}$ génération peut ne pas être celle d'agneaux de race pure ou Fi Romanov, ou d'agneaux de souche synthétique ne bénéficiant plus alors d'un éventuel effet d'hétérosis : dans ces conditions, le seuil en dessous duquel des problèmes de viabilité pourraient se poser, pourrait être modifié et constituer une contrainte complémentaire. Il faudrait alors être plus attentif au choix de la race de mâle de croisement terminal, tout comme accorder une attention particulière à la valeur maternelle des brebis dans la sélection de la souche synthétique comme le suggèrent déjà CROWLEY et MC GLOUGHIN (I972) à propos de croisements Finnois. Cependant si les conditions d'élevage sont favorables, et si en conséquence un plus faible niveau. de production laitière n'affecte pas la viabilité des agneaux, il est possible d'admettre une vitesse de croissance relativement plus faible jusqu'au sevrage et il serait alors plus intéressant de privilégier l'accroissement de la productivité numérique des brebis en vue d'obtenir globalement une productivité pondérale supérieure. 


\section{Remerciements}

Ces travaux n'ont pu être menés à bien qu'avec la contribution du personnel technique des troupeaux expérimentaux de La Fage et de Langlade que nous voulons remercier ici. Ce texte doit également beaucoup aux remarques et suggestions de nos collègues J. LABUSSrìrE et G. RICORDEAU et a bénéficié de l'aide du Service de Documentation du C.N.R.S. pour la traduction des publications russes.

\section{Summary}

\section{Evaluation of milking abilities of pure bred or crossbred Romanov ewes}

This study is concerned with the evaluation of the milk production potential of the Romanovbreed. Several types of comparisons are realised:

I. Experiment $I$. The milk production of purebred Romanov ewes with 3 lambs in suckling demonstrates a superiority of $73 \mathrm{p}$. I 00 by report to similar ewes with only I lamb reared (born from multiple lambing). Table 4. The daily yield is estimated by hormonal method. This increasing provided by higher lamb number in rearing is very similar to the observed one in other so different breeds as Chios or crossbred Blackface. The highest differences beetween the two ewes group (I and 3 lambs) is observed during the second week after lambing (fig. I $a$ ).

2. Experiment II. To eliminate the effect of the lamb number, the comparison beetween several genotypes of crossbred ewes Romanov $\times$ Berrichonne $d u$ Cher is realised only with ewes which lamb and rear 2 lambs. We observe a decreasing of the milk yield as the Romanov blood percentage increases in the genotype (table 8). It appears that differences have to be attributed to the ewes genotypes and not to the lambs genotypes which exteriorise at the less similar growth potential with upper Romanov blood observed upon twin lambs in artificial rearing or sucked as single (table ro). Similar results are reported about Finnish Landrace breed.

3. An other attempt to eliminate lamb influence is to do comparisons in milking. We observe less milk with Romanov ewes by report to Lacaune ewes. But problems of adaptation to machine milking can interfer in this comparison.

This results give a general figure of lower milk production potential for prolific breeds. They are discussed in the view of different crossing strategies in which Romanov and Finnish breed. They are used.

\section{Références bibliographiques}

ANTILA U., I975. The milk yield of Finnsheep ewes (en Finnois). Lammastalous I975 (4), I 5-21 . Lammastalous i976 (I), I3-16. Cité par TrEacher et GibB (I978).

Bonaiti B., Harraca E., I975. Résultats non publiés.

Brunel J. C., Lefente C., Tchamitchian L., 1975. Performances de reproduction et d'élevage de brebis Finnoises et Romanov en race pure. Ire Journées Rech. Ovine Caprine, I.N.R.A.I.T.O.V.I.C., SPEOC, Paris, 2, 3-17.

Crowl,Ey J. P., Mc Gloughlin P., I972. Milk production of Finnish Landrace sheep. Irish J. agric. Res., 11, II 7-1 19.

DESvignes A., I970. Étude préliminaire de l'effet d'hétérosis et de l'effet maternel sur le caractère de prolificité des brebis en croisement réciproque Romanov $\times$ Berrichon. I $\mathrm{z}^{\mathrm{e}}$ Session Commission Génétique F.F.Z., Gödöllö, Budapest, 24-28 août. Ann. Génét. Sél. anim., 3, roo.

DESVIGNES A., r97I. La race ovine Romanov. Revue bibliographique. Ann. Zootech., 20, 353-370. Donald H. P., Read J. L., Russel W. S., I968. A Comparation trial of crossbred ewes by Finnish Landrace and other sires. Anim. Prod., 10, 413-42I.

Flamant J. C., Jacouin Michèle, i975. Résultats non publiés. 
Flamant J. C., CASU S., 1977. Différences entre races pour le potentiel de production laitière et amélioration génétique de cette production. Rapport Commission Ovine Caprine $28^{\mathrm{e}}$ Réunion annuelle F.E.Z., Bruxelles, 22-25 août.

Flamant J. C., JacQuin M., MaQuere M., 1977. Quelques aspects génétiques de la vitalité chez les agneaux. $3^{\text {es }}$ Journées Rech. Ovine Caprine. I.N.R.A.-I.T.O.V.I.C., SPEOC, Paris, 200-2 I I.

Flamant J. C., Labussiere J., 1972. Premières observations sur les aptitudes laitières des brebis de race Romanov. Ann. Zootech., 21, 375-384.

GARDNER R. W., HoGue D. E., I 964 . Efffects of energy intake and number of lambs sukled on milk yield, milk composition and energetic efficiency of lactating ewes. J. anim. Sci., 23, 935-942.

GaRdner R. W., Hogue D. E., Bensadoun A., I964. Body composition and efficiency of growth of suckling lambs as affected by level of feed intake. J. anim. Sci., 23, 943-952.

Kalaissakis P., Papadimitriou T., Flamant J. C., Boyazoglu J. G., Zervas N., i 977. Comparaison des races ovines Chios et Frisonne avec leurs croisements en Grèce continentale. II. Production laitière. Ann. Génét. Sél. anim., 9, 181-201.

Kasimov K. M., I972. Élevage d'agneaux de race Romanov séparés des brebis (en Russe). Zhivotnovodstvo, 32 (7), 77-79.

Kovnerev I. P., Zamorychev A. V., Selianine G. I., Smirnov L. F., Iadrilev V. I., i967. Organisation et technique d'élevage des ovins Romanov. Kolos Edit., Moscou, 232 pp., cité par DESVIGNES (197I).

LABUSSIERE J., I979. Communication personnelle.

LABUSSIERE J., COMBaUd J. F., 1978. Effet du nombre journalier de traites et de tétées sur la production laitière des brebis $=$ interprétation physiologique. Symp. Traite méc. Petis Ruminants, Alghero, 22-27 mai 42-53.

MAGomedov I. M., I975a. Relation entre le poids des agneaux Romanov et la production laitière des mères (en Russe). Ovtsevodstvo (6), 32-34.

MAGomedov I. M., I975b. Quantité et composition du lait des brebis Romanov (en Russe). Ovtsevodstvo (2), 34-35.

Mc Clelland T. H., I975. About sheep breeding. Speeding up the creation of new breeds. Abro Report 1975, Edinburgh. Agric. Res. Council (1975), 5-9.

MOORE R. W., r966. Genetic factors affecting the milk intake of lambs. Austr. J. Agric. Res., 17, I9I-I99.

Peart J. N., Edwards R. A., Donaldoson E., 1972. The yield and composition of Finnish Landrace $\times$ Blackface ewes. J. agric. Sci., 79, 303-3 3 .

Piestrak T., Flamant J. C., Molenat G., I975. L'allaitement artificiel des agneaux. Quelques observations technico-économiques effectuées sur le troupeau du domaine de La Fage. Bull. techn. Centre Rech. Vét. Zoot. Theix (19), 37-44.

POUJARdieu B., I969. Recherche d'une méthode d'estimation de la production laitière des femelles ovines et bovines pendant la phase d'allaitement. Ann. Zootech., 18, 299-3I5.

RiCORDEAU G., BOCCARD R., I96I. Relations entre la quantité de lait consommé par les agneaux et leur croissance. Ann. Zootech., 10, I I3-125.

Ricordeau G., Razungles J., Eychenne F., Tchamitchian I., i976a. Performances de reproduction des brebis Berrichonne du Cher, Romanov et croisées. II. Composantes de la prolificité. Ann. Génét. Sél. anim., 8, 25-35.

Ricordeau G., Tchamitchian L., Eycheynne F., Razungles J., I976b. Performances de reproduction des brebis Berrichonne du Cher, Romanov et croisées. I. Activité sexuelle en début de saison et à contre saison. Ann. Génét. Sél. anim., 8, 9-24.

Ricordeau G., TChamitchian L., Lefevre C., Brunel, J. C., 1977. Différences génétiques de viabilité entre agneaux de bergerie Romanov, Berrichon du Cher, Fl. Romanov $\times$ Berrichon et Finnois. $3^{\mathrm{e}}$ Journées Rech. Ovine Caprine. I.N.R.A.-I.T.O.V.I.C., SPEOC Paris, I89-I99.

Ricordeau G., Tchamitchian L., Lefenre C., Brunel J. C., Desvignes A., I976. Amélioration de la productivité des brebis Berrichonne du Cher (BC) par croisement. III. Performances de reproduction des trois premières générations de croisées entre les races $\mathrm{BC}$ et Romanov. Ann. Génét. Sél. anim., 8, 405-4I9.

Ricordeau G., Tchamitchian L., Thimonier J., Flamant J. C., Theriez M., i978. First survey of results obtained in France on reproductive and maternal performance in sheep, with particular reference to the Romanov breed and crosses with it. Livestock Prod. Sci., 5, I 81-201.

Smrrnova V. Ia., I958. Production laitière des brebis Romanov. Trud. Vop. Kormodobyr. Svinovod. Ovtsevod., 3, 27I-339. Cité par Desvignes (197I). 
Sommer W., Morag M., Finger K. H., Wassmuth R., 1974. Lämmerwachstum und Milchleistung bei säugenden Deutschen Schwarzköpfigen Fleischs:hafen und Finnkreuzungen I, Milchmenge. Züchtungsk., 46, 27-35.

TCriamitchian L., i975. Stratégies d'utilisation des races prolifiques en race pure et en croisement. Ire Journées Rech. Ovine Caprine. I.N.R.A.-I.T.O.V.I.C., SPEOC Paris, 2, 82-102.

THERIEZ M., Tissier M., i975. L'utilisation des races prolifiques. Valeur d'élevage des animaux croisés et qualité des carcasses. $I^{r e}$ Journées Rech. Ovine Caprine. I.N.R.A.-I.'T.O.V.I.C., SPEOC Paris, 2, 64-8I.

TrEAcher T. T., GibB M. J., 1978. The milk yield of Finnish Landrace $\times$ Dorset. Horn ewes milked by machine. Symp. Traite méc. Petits Ruminants, Alghero, 22-27 mai, I I3-I 2 I.

YADRICHEV V. I., 1969. Effect of prolificacy in Romanov ewes on production characters in their progeny (en Russe). Sb. nouch. Trud. Ivanov. Sel. Khoz. Inst., (30), 95-100, d'après A.B.A., $39(4676)$. 